\title{
Analysis of Dynamic Spectrum Access Gaining Devices for Wireless Sensor Network: A Survey
}

\author{
Megha Thakre $^{1}$, N. Sahare ${ }^{2}$ \\ Department of Computer Science and Engineering, G.H Raisoni Institute of Engineering and Technology for Women, \\ Nagpur, Maharashtra, India ${ }^{1}$ \\ Assistant Professor, Department of Computer Science and Engineering, G.H Raisoni Institute of Engineering and \\ Technology for Women, Nagpur, Maharashtra, India ${ }^{2}$
}

\begin{abstract}
Cognitive radio is a communication technology which is designed to serve users to make use of the available bandwidth in a greatest possible amount. Though more research has been done on spectrum sensing and allocation problems, cognitive radio has recently attracted a lot of research interest. This paper shows the concept of cognitive radio networks (CRNs). Cognitive radio is an intelligent form of wireless communication. It consists of a transceiver which detects the channels which are in use for communication or which are not in use. It can automatically search vacant channels in a wireless spectrum. The cognitive radio depends on spectrum allocation and channel selection. This paper gives the survey on various methods for cognitive radio.
\end{abstract}

Keywords: Cognitive Radio Networks(CRNs), Spectrum allocation, Primary Users(PU), Secondary Users(SU).

\section{I.INTRODUCTION}

COGNITIVE radio networks (CRNs) have capability to improve the utilization of spectrum by making the good use of the vacant spectrum. For example IEEE 802.22 wireless regional area network (WRAN) standard enables devices to make use of TV bands for Internet access which is based on the cognitive radio (CR) concept. A Secondary User (SU) in a CRN accesses an unused spectrum at a particular time interval in an opportunistic manner which is a channel issued to a Primary User (PU). There are many DSA techniques exist among which channel assembling (CA) is a popular one whose target is increasing the ability of performance of the secondary network by permitting one SU to mass various idle available channels. The implementation of channel assembling is based on two ways depends on the idle channels available in the spectrum. In this paper two separate queues are allocated for the secondary users. The main aim of this paper is to give consideration to those blocked or dropped SU services by the strategy in a queue so that at a later instant they can finish their services [1].

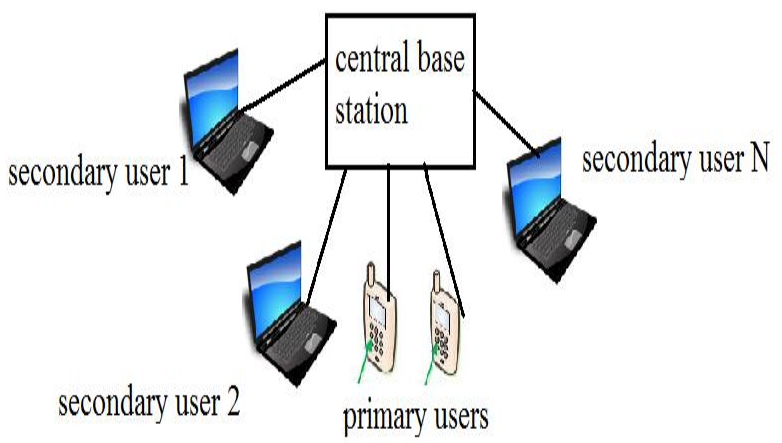

Fig1. Network scenario of a CRN

This paper proposes a new cooperative pattern for protective communication in cognitive radio networks
(CRNs) where secondary users (SUs) are permitted to access the spectrum licensed to the primary users (PUs) as long as they keep the secure communication of PUs in its original or existing state in the presence of harmful eavesdroppers. The security against the interference of the third parties is very critical problem in CRNs. To solve this problem, cooperative jamming [2] is introduced. With the increasing demand for the resources of the spectrum, many frequency bands such as unlicensed and licensed bands are crowded. Dynamic spectrum access (DSA) [3] is one of the essential components in the design of cognitive radio networks. It gives the idea of the centralized and the distributed DSA algorithms. Mainly there are two contributions: 1) The proposed algorithm performs the CFDSA (control free) algorithm by calculating the aggregate throughput and the traffic distribution fairness. 2) Present an analytical model to estimate the no SU collision with PU probability and the aggregate throughput of the proposed algorithm [3].

In cognitive radio networks (CRNs) where the authorized owners or primary operators (POs) could actively share their unused spectrum with secondary users (SUs), auction theory has been extensively used for spectrum allocation in CRNs to achieve the fairness and increase efficiency. In this theory, the coalition, that is, temporary group double auction for efficiently allocating the spectrum in CRNs is introduced where secondary users (SUs) are divided into several coalitions and the reusability of spectrum can be executed within each coalition [4].

In [5] a statistical estimation of the number of available channels for usage by secondary users opportunistically in a spectrum band is provided. It is obvious that the behaviour of the CR nodes depends on the occupancy patterns of the authorized users which vary as a function of frequency, time, geolocation, and other features. Therefore, accurate modelling of such occupancy patterns 
becomes absolutely compulsory in estimating the performance of the network as well as helps effectively design and execute efficient $\mathrm{CR}$ technologies with minimal interference. A systematic overview on $\mathrm{CR}$ communications and networking [6] by reviewing the key functions of the physical (PHY), medium access control (MAC), and network layers involved in a CR design.

\section{RELATED WORK}

INDIKA A. M. BALAPUWADUGE, LEI JiAO, ViCENT PlA AND FRANK Y. LI [1], proposed the queuing model designed with two queues. One of the queues is designed for incoming SUs and the other one is used for allocating resources of the bandwidth for both PU and SU services. The requests of both PU and SU are buffered inside the bandwidth allocation queue (BAQ). Primary users always have higher priority than the secondary users and therefore, the first come first served (FCFS) discipline does not apply to BAQ. The queuing discipline is designed in a manner that the waiting time of a real-time $\mathrm{SU}$ request is minimized so that delay-sensitive nature can be maintained.

Nader Mokari, Saeedeh Parsaeefard, Hamid Saeedi, and Paeiz Azmi [2], introduced a cooperative secure allocation of the resources in order to prevent the communications of the PUs from the malicious eavesdroppers. An interference limited CRN is considered in which a primary network with single transmitter and receiver exists. There is a trustworthy secondary network and a set of eavesdropping malicious nods which try to interfere and overhear the primary and secondary messages. The primary transmitter (PT) wants to send data confidentially to its corresponding receiver in its own available spectrum. Ming Zhao, Chang-chuan Yin, and Xiao-jun Wang [3], introduced the DSA algorithm without the use of control channel. In this strategy, a network having extremely heavy traffic load is considered. Considering heavy traffic each SU always has some data for transmission purpose to any other SU in the network. The time is divided into consecutive periods which are also known as the opportunistic spectrum periods (OSP) and therefore, the SUs work in time-slotted mode. There are four phases in each phase:

1) Selection of mode for working and formation of pair

2) Sensing of spectrum

3) Selection of channel

4) Transmission of data

Gaofei Sun, Xinxin Feng, Xiaohua Tian, Xiaoying Gan,

Youyun $\mathrm{Xu}$, Xinbing Wang, and Mohsen Guizani [4] derived the economically full spectrum reusable allocation among SUs for CRNs. Along with the coalition formation process the auction mechanism is incorporated and the coalitional double auction mechanism is developed. In the auction process there are several virtual auctions and a final auction. In virtual auctions, the SUs are partitioned into several coalitions by the auctioneer to achieve the optimization goal according to outcomes of the virtual auction. The process gives 3 -fold contribution:

1) Establishment of a novel and feasible framework

2) Proposing a coalition formation algorithm
3) Verifying the convergence of the proposed algorithm Amine Amich, Muhammad Ali Imran, Rahim Tafazolli and Parissa Cheraghi [5] proposed accurate techniques and algorithms following an independent and non-identical distribution (i.n.i.d.) paradigm for probabilistic availability of spectrum modelling. The problem of describing the number of channels that are available for usage by $\mathrm{CR}$ users in a spectrum band in an opportunistic manner is analyzed with the advantage of the probability of a channel being free. With this information preferable sections of the spectrum are selected for further sensing. Table 1 illustrates a comparison of the algorithms running time given the input $n$ is varied.

TABLE 1

COMPARISON OF ALGORITHMS

\begin{tabular}{|c|c|c|c|c|}
\hline Algorithm & $\mathrm{n}=32$ & $\mathrm{n}=64$ & $\mathrm{n}=256$ & $\mathrm{n}=512$ \\
\hline Conventional & 482 & 2866 & NA & NA \\
\hline M-conv $(m=4)$ & 0.015 & 0.046 & 0.14 & 0.52 \\
\hline Recursive & 0.0001 & 0.0005 & 0.002 & 0.015 \\
\hline Hybrid $(m=8)$ & 0.0003 & 0.0006 & 0.001 & 0.006 \\
\hline Poisson-normal & 3.18 & 9.7 & 3028 & NA \\
\hline Camp-Paulson & 0.012 & 0.098 & 0.81 & 1.93 \\
\hline
\end{tabular}

Ying-Chang Liang, Kwang-Cheng Chen, Geoffrey Ye Li, and Petri Mähönen [6], addressed the signal processing techniques for spectrum sensing, cooperative spectrum sensing, and transceiver design for cognitive spectrum access for the PHY layer and reviewed the sensing scheduling schemes, sensing-access trade-off design, spectrum aware access MAC, and selected CR MAC protocols for the MAC layer.

\section{PROPOSED SYSTEM}

In order to gain the access through any available medium, the proposed system will enable the network selfmanagement by using autonomic computing where device can switch to available network on demand. In this proposed system, primary users have the full authority of accessing their allocated frequency bands whereas secondary users can make use of channels which are not occupied by the PUs in an opportunistic manner. The main aim of the proposed system is to achieve the break less communication in the network. This will make the quality of mobile node efficient in the network when it is travelled from one service provider to another service provider. The proposed system will be worked out in four phases as follows:

Phase 1: Discovery of available network

Phase 2: For the connection of other network, switching the network automatically

Phase 3: Maintain load balancing in base station reliably Phase 4: Improvement of coverage capacity

Figure 2 shows tower and a wireless node where a node is requesting for the access to the tower and tower is sending back the acknowledgment as accepting the request. 


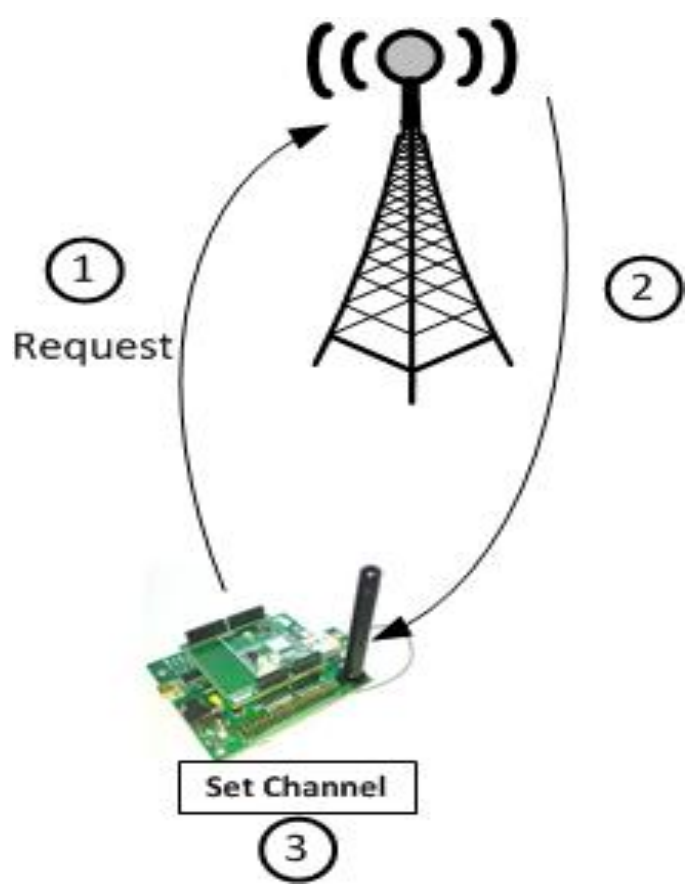

Fig2. A tower and a wireless node

\section{CONCLUSION}

The proposed system will be a priority queue based channel assembling strategy. The heterogeneous system for handoff process between spectrum and base station will be used. The heterogeneous network is better for the proposed system because the heterogeneous network combine several different networks. The proposed system will be a feasible self configurable device. In the proposed system, each SU will independently sense multiple data channels. The proposed system will be used to design for cognitive radio implementation.

\section{REFERENCES}

1] Indika A. M. Balapuwaduge, Lei Jiao, Vicent Pla, and Frank Y. Li, "Channel Assembling with Priority-Based Queues in Cognitive Radio Networks:Strategies and Performance Evaluation”, IEEE transactions on wireless communications, vol. 13, no. 2, February 2014

[2] Nader Mokari, Saeedeh Parsaeefard, Hamid Saeedi, and Paeiz Azmi, "Cooperative Secure Resource Allocation in Cognitive Radio Networks with Guaranteed Secrecy Rate for Primary Users", IEEE transactions on wireless communications, vol. 13, no. 2, February 2014.

[3] Ming Zhao, Chang-chuan Yin, and Xiao-jun Wang, "A Novel Dynamic Spectrum Access Algorithm for Cognitive Radio Networks", JOURNAL OF COMMUNICATIONS AND NETWORKS, VOL. 15, NO. 1, February 2013.

[4] Gaofei Sun, Xinxin Feng, Xiaohua Tian, Xiaoying Gan, Youyun $\mathrm{Xu}$, Xinbing Wang, and Mohsen Guizani, "Coalitional Double Auction for Spatial Spectrum Allocation in Cognitive Radio Networks", IEEE transactions on wireless communications, vol. 13, no. 6, June 2014

[5] Amine Amich, Muhammad Ali Imran, Rahim Tafazolli and Parissa Cheraghi, "Accurate and Efficient Algorithms for Cognitive Radio Modeling Applications under the i.n.i.d. Paradigm", IEEE transactions on vehicular technology, July 2014

[6] Ying-Chang Liang, Kwang-Cheng Chen, Geoffrey Ye Li, and Petri Mähönen, "Cognitive Radio Networking and Communications: An Overview," IEEE transactions on vehicular technology, vol. 60, no. 7, September 2011. 